\title{
ON BOUNDEDNESS OF FRACTIONAL MAXIMAL OPERATOR IN WEIGHTED $L^{p(.)}$ SPACES
}

\author{
FARMAN I. MAMEDOV AND YUSUF ZEREN
}

Abstract. In this paper, we derive some sufficient conditions for the boundedness of the fractional maximal operator in the weighted variable exponent Lebesgue spaces $L^{p(.)}$, where Sawyer's type pair of modular conditions are proposed on a weight functions and it is assumed a local logregularity and a decay condition on the exponent function $p($.$) .$

Mathematics subject classification (2010): 42A05, 42B25, 26D10, 35A23.

Keywords and phrases: Fractional maximal operator, weighted Lebesgue space, variable exponent.

\section{REFERENCES}

[1] M. Asif, V. Kokilashvili, AND A. Meskhi, Boundedness criteria for maximal functions and potentials on the half-space in weighted Lebesgue spaces with variable exponent, Int. Trans. Spec. Funct. 20 (2009), 805-819.

[2] C. CApone, D. CRuZ-URibe SFO AND A. Fiorenza, The fractional maximal operator on variable $L^{p}$ spaces, Revista Math. Iberoamericana. 23, 3 (2007), 747-770.

[3] D. CruZ-Uribe, L. Diening, A. Fiorenza, A new proof of the boundedness of the maximal operator on variable Lebesgue spaces, Bol. Unione Mat. Ital. (9) 2, 1 (2009), 151-173.

[4] D. Cruz-Uribe, A. Fiorenza And C. J. Neugebauer, The maximal function on variable $L^{p}$ spaces, Ann. Acad. Scient. Fenn. Mathematics. 28 (2003), 223-238.

[5] D. CRUZ-URIBE, New proofs of two-weight norm inequalities for a maximal operator, Georgian Math. Journal. 7, 1 (2000), 33-42.

[6] D. Cruz-Uribe, A. Fiorenza And C. J. Neugebauer, Weighted norm inequalities for the maximal operator on variable Lebesgue spaces, J. Math. Anal. Appl. 394 (2012) 744-760.

[7] D. Cruz-Uribe, A. Fiorenza, Variable Lebesgue Spaces. Foundations and Harmonic Analysis, Series: Applied and Numerical Harmonic Analysis, Birkhäuser, Basel, 2013, IX, 312 p.

[8] L. DiEning AND P. Hasto, Muckenhoupt weights in variable exponent spaces, Preprint, 2010.

[9] L. DiEnIng, Maximal functions on Musielak-Orlicz spaces and generalized Lebesgue spaces, Bulletin des Sciences Mathématiques 129, 8 (2005), 657-700.

[10] L. Diening, Maximal function on generalized Lebesgue spaces $L^{p(.)}$, Math. Inequal. Appl. 7, 2 (2004), 245-253.

[11] D. E. Edmunds, V. M. Kokilashvili And A. Meskhi, Two-weight estimates in $L^{p(x)}$ spaces with applications to Fourier series, Houston J. Math. 35, 2 (2009), 665-689.

[12] J. GarCiA-Cuerva AND J. M. MARTElL, Two-weight norm inequalities for maximal operators and fractional integrals on non-homogeneous spaces, Indiana Univ. Math. J. 50, 3 (2001), 1241-1280.

[13] A. HaRman AND F. I. MAMEDOv, On boundedness of weighted Hardy operator in $L^{p(.)}$ and regularity condition, J. Ineq. Appl. 2010, 2010:837951 (6 December 2010)

[14] P. Harjulehto, P. HÄstö And M. Pere, Variable exponent Lebesgue spaces on metric spaces: the Hardy-Littlewood maximal operator, Real Anal. Exchange. 30 (2004-2005), 87-104.

[15] V. M. KoKILAshVILI AND A. MESKHI, Weighted criteria for generalized fractional maximal functions and potentials in Lebesgue spaces with variable exponent, Integral Transforms and Special Functions, 18, 9 (2007), 609-628

[16] V. M. KokilashVili And A. Meskhi, Two-weight inequalities for fractional maximal functions and singular integrals in $L^{p(.)}$ spaces, J. Math. Sci. 173, 6 (2011), 656-673. 
[17] V. M. KokilashVili And S. G. SAmKo, Operators of harmonic analysis in weighted spaces with non-standard growth, J. Math. Anal. Appl. 352, 1 (2009), 15-34 (2008).

[18] V. KokilashVili AND S. SAMKO, Boundedness of maximal operators and potential opertators on Carleson curves in Lebesgue spaces with variable exponent, Acta Mathematica Sinica, 24, 11 (2008), 1775-1800.

[19] V. KoKILASHVIli AND S. SAMKO, The maximal operator in weighted variable exponent spaces on metric spaces, Georgian Math. J. 15 (2008), p. 683-712.

[20] V. M. Kokilashvili and S. G. Samko, The maximal operator in weighted variable spaces on metric spaces, Proc. A. Razmadze Math. Inst., 144 (2007), 134-137.

[21] V. M. Kokilashvili, S. G. Samko and N. Samko, The maximal operator in weighted variable spaces $L^{p(.)}$, Journal of Function Spaces and Applications, 5 (2007), 3, 299-317.

[22] V. M. Kokilashvili And S. G. Samko, Maximal and fractional operators in weighted $L^{p(x)}$ spaces, Revista Mathematica Iberoamericana, 20, 2 (2004), 493-515.

[23] O. KovaciK And J. Rakosnik, On spaces $L^{p(x)}$ and $W^{L^{p(x)}}$, Czechoslovak Math. J. 41 (116) (1991), 592-618.

[24] A. K. LERnER, Some remarks on the Hardy-Littlewood maximal function on variable $L^{p}$ spaces, Math. Z. 241, 3 (2005), 509-521.

[25] F. I. MAMEdov AND Y. ZEREN, On a two weighted estimation of maximal operator in the Lebesgue space with variable exponent, Anali di Matematica, 190, 2 (2010), 263-275.

[26] F. I. MAMEDOV AND Y. ZEREN, Two-weight inequalities for the maximal operator in a Lebesgue space with variable exponent, J. Math. Sci. 173, 6 (2011), 701-716.

[27] A. NeKVIndA, Hardy-Littlewood maximal operator on $L^{p(.)}\left(R^{n}\right)$, Math. Inequal. Appl., 7, 2 (2004), $255-265$.

[28] L. PICK AND M. RUZICKA, An example of a space $L^{p(x)}$ on which the Hardy-Littlewood maximal operator is not bounded, Expo. Math. 19, 4 (2001), 369-371.

[29] Y. Rakotondratsimba, On Muckenhoupt and Sawyer conditions for maximal operators, Publ. Mat. 37 (1993), 57-73.

[30] E. T. SAWYER, A characterization of a two-weight norm inequality for maximal operators, Studia Math. 75 (1982), 1-11.

[31] S. S AMKO, Convolution type operators in $L^{p(x)}$, Integr. Transf. and Spec. Funct, 7, 1-2 (1998), 123144. 